\title{
BRAP2 inhibits the Ras/Raf/MEK and PI3K/Akt pathways in leukemia cells, thereby inducing apoptosis and inhibiting cell growth
}

\author{
HIROHARU SAKAI $^{1}$, ISAMU SHIINA ${ }^{2}$, TAKAHISA SHINOMIYA ${ }^{1}$ and YUKITOSHI NAGAHARA ${ }^{1}$ \\ ${ }^{1}$ Division of Materials and Life Sciences, Graduate School of Advanced Science and Technology, \\ Tokyo Denki University, Hatoyama, Saitama 350-0394; ${ }^{2}$ Department of Applied Chemistry, \\ Faculty of Science, Tokyo University of Science, Shinjuku-ku, Tokyo 162-8601, Japan
}

Received May 26, 2020; Accepted December 8, 2020

DOI: $10.3892 /$ etm.2021.9894

\begin{abstract}
Breast cancer susceptibility gene 1 (BRCA1)associated protein 2 (BRAP2) is a novel protein that binds to BRCA1 and is located in the cytoplasm. BRAP2 has been demonstrated to bind to regulators of the Ras-Raf-MEK and PI3K/Akt pathways, both of which are involved in carcinogenesis. This suggests that BRAP2 may be capable of regulating both pathways. In the present study, the role of BRAP2 in both pathways was clarified during apoptosis and cell proliferation in a leukemia cell line. A BRAP2-deficient leukemia cell line was generated using CRISPR/Cas9, the BRAP2-deficient and parental cells were treated with a Ras, pan-Raf or PI3K inhibitor, and the changes in signal transduction, apoptosis and cell proliferation were evaluated. BRAP2 knockout attenuated the inhibition of signal transduction of the Ras-Raf-MEK and PI3K/Akt pathways by the Ras, pan-Raf or PI3K inhibitor. BRAP2 deletion also suppressed the cytotoxic and apoptotic effects of the Ras and pan-Raf inhibitors. However, the loss of BRAP2 did not suppress the cytotoxicity of the PI3K inhibitor but did suppress the PI3K inhibitor-induced inhibition of cell proliferation. The present results indicated that BRAP2 induces apoptosis and the inhibition of cell proliferation via regulating the Ras-Raf-MEK and PI3K/Akt pathways. In leukemia cells, because the Ras-Raf-MEK and PI3K/Akt pathways are activated aberrantly, the simultaneous inhibition of both pathways is desired. The current results indicated that enhancement of the function of BRAP2 may represent a new target in leukemia treatment.
\end{abstract}

Correspondence to: Professor Yukitoshi Nagahara, Division of Materials and Life Sciences, Graduate School of Advanced Science and Technology, Tokyo Denki University, Hiki-gun, Hatoyama, Saitama 350-0394, Japan

E-mail: yuki@mail.dendai.ac.jp

Key words: breast cancer susceptibility gene 1-associated protein 2, Ras-Raf-MEK pathway, PI3K/Akt pathway, apoptosis, cell cycle arrest, leukemia

\section{Introduction}

The Ras-Raf-MEK pathway is a mitogen-activated protein kinase (MAPK) pathway. Ras is activated by growth factors such as epidermal growth factor (EGF), then further activates Raf, MEK, and ERK, which in turn promote cell proliferation $(1,2)$. Ras and raf have been reported as the most frequently mutated genes in cancers. For example, mutations of the ras gene occur in about $90 \%$ of pancreatic cancers and $~ 50 \%$ of colon cancers. Also, mutations of the raf gene occur in about $70 \%$ of melanomas and about $10 \%$ of colon cancers (3).The mutated Ras protein reduces GTPase function, becomes locked in a permanently activated state, and continues to send signals downstream (4). The mutated Raf protein also activates ERK through downstream MEK (5). ERK activated by mutated Ras or Raf promotes cell proliferation and enhances EGFR ligand expression, leading to hyperactivation of the Ras-Raf-MEK pathway. This excessive signal transduction plays role in carcinogenesis, cancer growth, and drug resistance (6,7). In the signal transduction of the Ras-Raf-MEK pathway, a scaffold protein called kinase suppressor of Ras (KSR) is also important. KSR promotes the complex formation of Raf,MEK, and ERK, thereby enhancing signal transduction (8-10). Thus, the inhibition of KSR suppresses the signal transduction of the Ras-Raf-MEK pathway and is useful as an anti-cancer treatment $(11,12)$.

In addition, it has been reported that Ras activates the phosphatidylinositol-3-kinase (PI3K)/Akt pathway $(13,14)$. Like the Ras-Raf-MEK pathway, the PI3K/Akt pathway is also involved in promoting cell proliferation and suppressing cell death (15-17). Therefore, in anti-cancer treatment, a drug that inhibits only the Ras-Raf-MEK pathway will not completely eradicate cancer if the PI3K/Akt pathway remains activated (18). Thus, because of its roles in both pathways, Ras has been considered the most important target protein in cancer treatment (19). However, due to the multi-functionality of Ras, numerous Ras inhibitor candidates failed to show an anti-cancer effect, and Ras has been the most difficult target for anti-cancer treatment (20). Now, a number of inhibitors inhibit downstream of the Ras pathway, but it is desirable to develop drugs that inhibit the Ras-Raf-MEK and PI3K/Akt pathways simultaneously (18). 
Breast cancer susceptibility gene 1 (BRCA1)-associated protein 2 (BRAP2) was identified as a novel cytoplasmically localized protein that binds to BRCA1 (21). It was later reported that BRAP2 not only binds BRCA1, but also functions as a cytoplasmic retention protein for $\mathrm{p} 21$ and $\mathrm{NF}-\kappa \mathrm{B}(22,23)$. Various studies revealed that BRAP2 plays role in diseases caused by myocardial infarction, carotid atherosclerosis and inflammation (23-25). Taken together, these reports suggest that BRAP2 affects various types of intracellular signals. Yeast two-hybrid screening revealed that BRAP2 interact with Ras. BRAP2 is reported to inhibit the Ras-Raf-MEK pathway by binding to KSR (26). Since the inhibition of complex formation by the binding of BRAP2 to KSR is an event downstream of Ras, BRAP2 may suppress the signal transduction of the Ras-Raf-MEK pathway irrespective of the presence of Ras mutations. Moreover, BRAP2 has been reported to bind to proteins other than KSR. One of them is a phosphatase protein known as $\mathrm{PH}$-domain and leucine-rich repeat protein phosphatase 1 (PHLPP1) (27). This protein is involved in Akt activation, and the regulation of PHLPP1 leads to inhibition of the PI3K/Akt pathway $(28,29)$. That is, BRAP2 may inhibit both the Ras-Raf-MEK pathway and the PI3K/Akt pathway to suppress the proliferation or induce the death of cancer cells.

However, much remains unclear about the relationship between BRAP2 and the Ras-Raf-MEK pathway in cancer cells, and the relation between BRAP2 and the PI3K/Akt pathway is even less clear. In this study, to investigate the functions of BRAP2 against the Ras-Raf-MEK and PI3K/Akt pathways, we treated cells of a BRAP2-deficient cell line with inhibitors of either pathway and evaluated the changes in signal transduction, apoptosis, and cell proliferation.

\section{Materials and methods}

Cells. Jurkat cells were purchased from DS Pharma Biomedical. THP-1 was provided by Dr Y. Kobayashi of Toho University (Chiba, Japan). BALL-1, HL-60 and MOLT-4F were provided by the Cell Resource Center for Biomedical Research, Tohoku University (Sendai, Japan).

Reagents. The farnesyl transferase inhibitor tipifarnib and the PI3K inhibitor LY294002 were purchased from Adooq Bioscience. The pan-Raf inhibitor LY3009120 was purchased from Selleck Chemicals. The PKC inhibitor staurosporine was purchased from Cayman Chemical. All inhibitors were solubilized in dimethyl sulfoxide (DMSO, Wako). Phorbol 12-myristate 13-acetate (PMA) was purchased from Wako.

Medium and cell culture. The cells were cultured in RPMI1640 medium (Sigma-Aldrich; Merck KGaA) containing $3.5 \mu \mathrm{g} / 1$ 2-mercaptoethanol (Wako), $75 \mathrm{mg} / \mathrm{l} \mathrm{kanamycin} \mathrm{sulfate} \mathrm{(Wako),}$ and $2 \mathrm{~g} / 1 \mathrm{NaHCO}_{3}$ (Wako) supplemented with $10 \%$ fetal bovine serum (Biofill) and maintained at $37^{\circ} \mathrm{C}$ in a humidified chamber (ESPEC) under an atmosphere of $95 \%$ air and $5 \% \mathrm{CO}_{2}$.

Antibodies. Anti-BRAP2 polyclonal antibody (cat. no. ab77721) was purchased from Abcam. Anti-KSR polyclonal antibody (cat. no. AP7202a) was purchased from WuXi AppTec (Shanghai, China). Anti- $\beta$-actin polyclonal antibody (cat. no. 4967S), anti-phosphorylated (p)-Raf monoclonal antibody (cat. no. 9427S), anti-Raf polyclonal antibody (cat. no. 9422S), anti-MEK polyclonal antibody (cat. no. 9122S), p-ERK polyclonal antibody (cat. no. 9101S), anti-ERK polyclonal antibody (cat. no. 9102S), anti-p-Akt polyclonal antibody (cat. no. 9271S), anti-rabbit IgG-HRP linked antibody (cat. no. 7074S) and anti-mouse IgG-HRP linked antibody (cat. no. 7076S) were purchased from Cell Signaling Technology. Anti-caspase-3 monoclonal antibody (cat. no. sc-7272), anti-p-MEK monoclonal antibody (cat. no. sc-81503) and anti-Akt polyclonal antibody (cat. no. sc-8312) were purchased from Santa Cruz Biotechnology.

Knockout of BRAP2 by CRISPR/Cas9.pSpCas9(BB)-2A-Puro (PX459) V 2.0 (Plasmid \#62988) was purchased from Addgene (30). The $B b s I$ site of the plasmid was cut with $B b s I$ (New England Biolabs) at $37^{\circ} \mathrm{C}$ for $1 \mathrm{~h}$. The gRNA of BRAP2 (top: CACCGGAAAGGCGCTGCGTTCGAAA, bottom: AAACTTTCGAACGCAGCGCCTTTCCC) designed in CRISPRdirect (https://crispr.dbcls.jp) was ligated to the $B b s I$ site of the plasmid using a DNA ligation kit (Takara Bio) at $16^{\circ} \mathrm{C}$ for $3 \mathrm{~h}$. The plasmid was transfected into Jurkat cells by using the Neon transfection system (Thermo Fisher Scientific, Inc.) under the conditions of pulse voltage 1350 (V), pulse width 10 (ms) and pulse number 3. Before this transfection, the Jurkat cells were cultured in serum-free RPMI1640 medium containing an antibiotic agent at $37^{\circ} \mathrm{C}$ for $4 \mathrm{~h}$. The cells $\left(2 \times 10^{6}\right)$ were washed with $\mathrm{Ca}^{2+}$ and $\mathrm{Mg}^{2+}$-free phosphate buffered saline (PBS), then supplemented with $30 \mu \mathrm{l}$ of resuspension buffer $\mathrm{R}$ (Thermo Fisher Scientific, Inc.) and $10 \mu \mathrm{l}$ of the plasmid DNA. After transfection, the transfected Jurkat cells were cultured in serum containing antibiotic-free RPMI-1640 medium containing 10\% FBS. After $72 \mathrm{~h}$, the transfected Jurkat cells were cultured in RPMI-1640 medium containing $10 \% \mathrm{FBS}$ and $0.5 \mu \mathrm{g} / \mathrm{ml}$ puromycin (Sigma-Aldrich; Merck KGaA) for one month. For single cell cloning, the drug-selected Jurkat cells were diluted and seeded at 1 cell per well in 96-well plates (Becton, Dickinson and Company). The wells were confirmed to each have a single cell by examination under a phase contrast microscope (Olympus) every 2 days. As soon as they began to grown, single cell clones were transferred to 24-well (Sigma-Aldrich; Merck KGaA), 12-well (Corning) or 6-well (Thermo Fisher Scientific, Inc.). Finally, stable strains of Jurkat (Mock) and Jurkat ( $\triangle$ BRAP2) cell lines were generated.

MTT assay. Cells $\left(2 \times 10^{4}\right)$ were incubated in 96-well plates with or without the Ras, pan-Raf or PI3K inhibitors at $37^{\circ} \mathrm{C}$ for $24 \mathrm{~h}$. At $1 \mathrm{~h}$ prior to the end of incubation, $10 \mu \mathrm{l}$ of $5 \mathrm{mg} / \mathrm{ml}$ MTT (Wako) solution was added to each well, and the plates were further incubated for $1 \mathrm{~h}$. Then, the 96-well plates were centrifuged at $300 \mathrm{~g}$ for $5 \mathrm{~min}$, and the supernatant of each well was removed. One-hundred microliters of DMSO was added to each well, and the cell viability was determined by measuring the absorbance of the formazan at $570 \mathrm{~nm}$ using a microplate reader (Awareness Technology).

SubG1 and cell cycle analysis by propidium iodide staining. Cells $\left(2 \times 10^{6}\right)$ were treated with the Ras, Pan-Raf or PI3K 
inhibitors at $37^{\circ} \mathrm{C}$ for $24 \mathrm{~h}$. The concentration of cells was adjusted to $1 \times 10^{6}$ cells and then the cells were washed with PBS. The cells were added to a solution containing $500 \mu \mathrm{l}$ of $0.1 \%$ Triton X-100 (Wako)-PBS, $5 \mu \mathrm{l}$ of $5 \mathrm{mg} / \mathrm{ml}$ RNase A (Wako), and $12.5 \mu \mathrm{l}$ of $1 \mathrm{mg} / \mathrm{ml}$ PI (Wako). After the cells were left in the dark for $20 \mathrm{~min}$ at room temperature, they were passed through a pore-size nylon mesh. PI fluorescence was measured by a flow cytometer (Becton, Dickinson and Company).

Measurement of cell proliferation rate. Cells $\left(1 \times 10^{6}\right)$ were seeded in a BioLite $60 \mathrm{~mm}$ tissue culture dish (Thermo Fisher Scientific, Inc.). The cells were counted at the indicated times with a counting chamber (Hirschmann). For the measurement of cell growth inhibition by the PI3K inhibitor, cells $\left(1 \times 10^{6}\right)$ were seeded in a BioLite $60 \mathrm{~mm}$ tissue culture dish, treated with the PI3K inhibitor at $37^{\circ} \mathrm{C}$ for $48 \mathrm{~h}$, and counted with a counting chamber.

SDS-PAGE and western blotting. Cells $\left(2 \times 10^{6}\right)$ were treated with drugs at $37^{\circ} \mathrm{C}$ for the indicated times. The cells were harvested and washed with PBS. Lysis buffer (50 mM HEPES, $\mathrm{pH} 7.5,150 \mathrm{mM} \mathrm{NaCl}, 10 \%$ glycerol, $1 \%$ Triton X-100, $1.5 \mathrm{mM}$ $\mathrm{MgCl}_{2}, 1 \mathrm{mM}$ EGTA, $1 \mathrm{mM}$ sodium orthovanadate, and $1 \%$ protease inhibitor cocktail; Sigma-Aldrich; Merck KGaA) was added to the cells, and the mixture was left on ice for $20 \mathrm{~min}$. The cells were centrifuged at $14,500 \mathrm{~g}$ for $15 \mathrm{~min}$, and the supernatants were harvested as lysate samples. The samples were quantified by a BCA protein assay (Takara Bio) and adjusted to $2 \mathrm{mg} / \mathrm{ml}$ using lysis buffer. After the addition of a sample application buffer (4\% SDS, $125 \mathrm{mM}$ Tris, $\mathrm{pH} 6.8$, $10 \%$ glycerol, $0.02 \mathrm{mg} / \mathrm{ml}$ bromophenol blue, $10 \%$ 2-mercaptoethanol), the samples were heated at $100^{\circ} \mathrm{C}$ for $3 \mathrm{~min}$. SDS-PAGE was performed on $4 \%$ concentrated gel and $12 \%$ or $15 \%$ running gel. After the protein was transferred to a polyvinylidene di-fluoride (PVDF) membrane (Bio-Rad), the PVDF membrane was soaked in 3\% skim milk (Yukijirushi) for $1 \mathrm{~h}$ at room temperature. The skim milk dilution was changed and the membrane was soaked twice more for $30 \mathrm{~min}$ each at room temperature. The PVDF membrane was probed with the indicated primary antibodies $(1: 1,000)$ at $4^{\circ} \mathrm{C}$ overnight. The primary antibodies were collected, and the PVDF membrane was washed with $0.1 \%$ Tween-20 (Wako)-PBS. The PVDF membrane was soaked in either anti-rabbit IgG-HRP-linked antibody (Cell Signaling Technology) or anti-mouse IgG-HRP-linked antibody (Cell Signaling Technology) $(1: 2,000)$ for $1 \mathrm{~h}$ at room temperature. The secondary antibodies were washed out and the PVDF membrane was washed three times with $0.1 \%$ Tween-20-PBS for $15 \mathrm{~min}$ per wash. The PVDF membrane was soaked in ECL Western Blotting Substrate (Thermo Fisher Scientific, Inc.) for $1 \mathrm{~min}$, then imaged with an ImageQuant LAS 4000 (GE Healthcare). For the observation of other proteins, in case of observing other proteins, the primary and secondary antibodies were stripped after imaging the PVDF membrane. Stripping was accomplished by soaking the PVDF membrane in stripping buffer (100 mM 2-mercaptoethanol, 2\% SDS and $62.5 \mathrm{mM}$ Tris, $\mathrm{pH} 6.7$ ) in $65^{\circ} \mathrm{C}$ water bath for $30 \mathrm{~min}$, then washing with $0.1 \%$ Tween-20-PBS for $15 \mathrm{~min}$. The PVDF membrane was soaked in skim milk and reprobed with another antibody. The relative density of bands was quantified using ImageJ software (National Institutes of Health).

Statistical analysis. All data are presented as the mean \pm SD of three experiments, and the statistical analyses were performed using Microsoft Excel for mac ver. 16.0 (Microsoft Corporation) and $\mathrm{R}$ ver. 4.0 software ( $\mathrm{R}$ Foundation for Statistical Computing). The Student's t-test was used to compare paired groups. One-way analysis of variance was used for multi-group analysis, followed by Bonferroni test as a post hoc test. $\mathrm{P}<0.05$ was considered to indicate a significant difference.

\section{Results}

$B R A P 2$ regulated the signal transduction of the Ras-Raf-MEK and PI3K/Akt pathways. First, to investigate the role of BRAP2 in the Ras-Raf-MEK and PI3K/Akt pathways, we knockout BRAP2 expression using CRISPR/Cas9. After transfecting the mock and designed BRAP2 knockout plasmids, we performed western blotting to determine whether BRAP2 was knocked out in Jurkat cells. The result showed that BRAP2 was knocked out in Jurkat ( $\triangle$ BRAP2) cells (Fig. 1A). Because BRAP2 was reported to be a cell cycle regulator (31), we measured the proliferation of Jurkat (Mock) and Jurkat ( $\triangle$ BRAP2) cells. We found that Jurkat $(\triangle$ BRAP2) cells proliferated more slowly than the parental cells (Fig. 1B), but the BRAP2 deletion did not induce cell death (data not shown). In addition, we conducted western blotting to evaluate how the loss of BRAP2 affected the Ras-Raf-MEK and PI3K/Akt pathways. The results showed that the levels of p-Raf, p-MEK and p-Akt were markedly higher in Jurkat ( $\triangle$ BRAP2) cells compared to Jurkat (Mock) cells, but the level of p-ERK was markedly lower in the Jurkat ( $\triangle$ BRAP2) cells. On the other hand, there was no change in the expression of KSR (Fig. 1C). These results suggest that BRAP2 knockout dose not directly downregulate or upregulate the Ras-Raf-MEK pathway to a significant degree, which is consistent with a previous study (26). Next, western blotting was performed to evaluate the effects of the loss of BRAP2 on the Ras, pan-Raf, and PI3K inhibitors. In Jurkat (Mock) cells, the levels of p-Raf, p-MEK, and p-ERK were decreased by treatment with the farnesyl transferase inhibitor tipifarnib, which is used as a Ras inhibitor, or with the pan-Raf inhibitor LY3009120. Conversely, in the Jurkat ( $\Delta$ BRAP2) cells, the levels of these phosphorylations were not decreased by Ras and pan-Raf inhibitors treatment (Fig. 1D). LY294002, a PI3K inhibitor, markedly inhibited the phosphorylation of Akt within $24 \mathrm{~h}$, but this was restored by $48 \mathrm{~h}$ treatment in Jurkat (Mock) cells. PI3K inhibitor did not decrease the level of p-Akt in Jurkat ( $\triangle$ BRAP2) cells (Fig. 1E).

Loss of BRAP2 suppressed apoptosis by Ras and pan-Raf inhibitors. BRAP2 has been shown to bind to KSR, thereby inhibiting the Ras-Raf-MEK pathway (26), and it also binds to PHLPP, which is a modulator of Akt $(28,29)$. In our study, because BRAP2 deletion suppressed the inhibition of signal transduction by the Ras and Pan-Raf inhibitor (Fig. 1D), we predicted that BRAP2 deletion also affects the cytotoxicity of these inhibitors. To investigate this possibility, we performed 
A

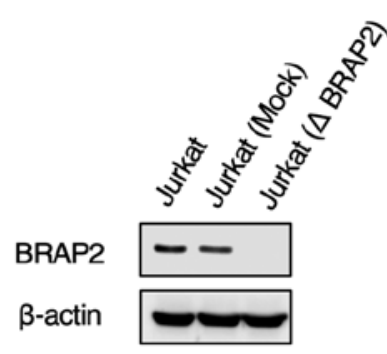

C

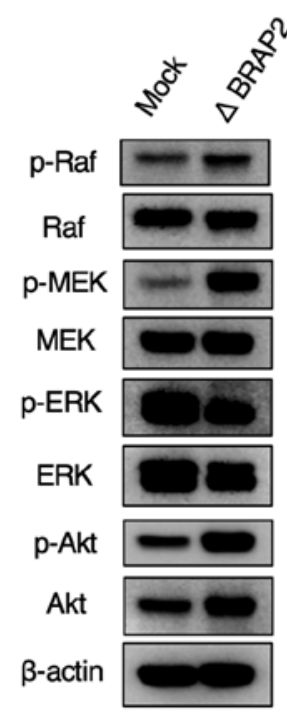

E
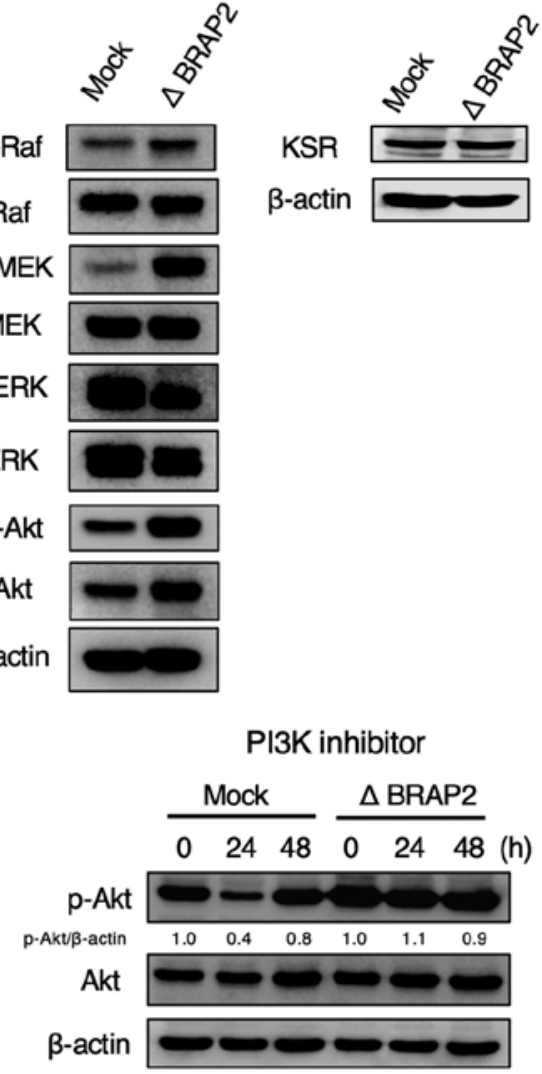

B

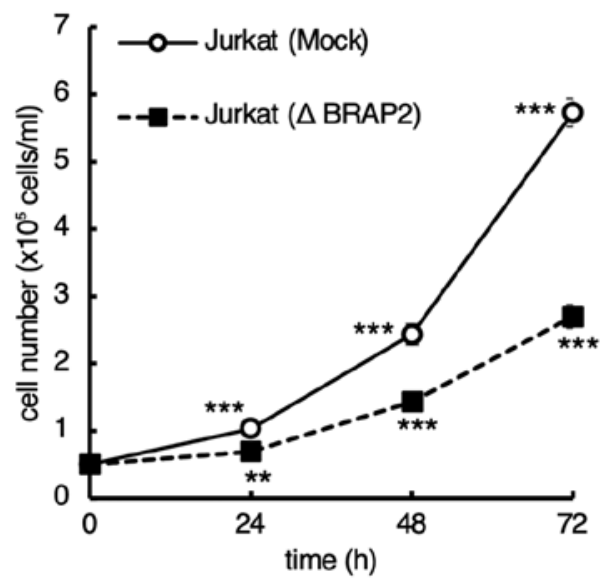

D

p-Raf

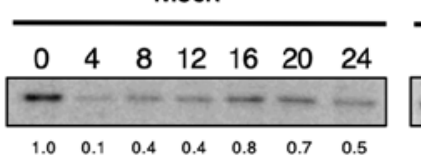

$\triangle B R A P 2$

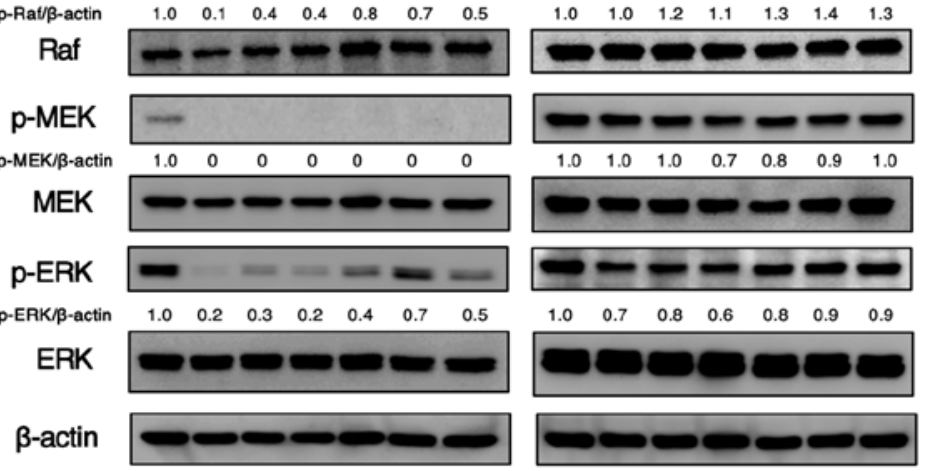

pan-Raf inhibitor

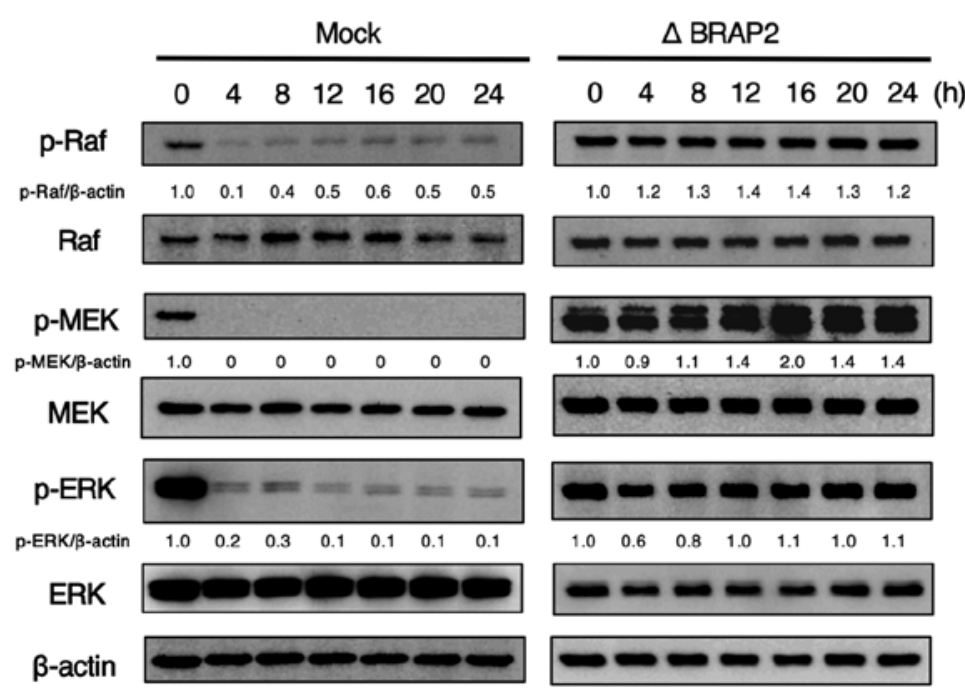

Figure 1. BRAP2 regulates the signal transduction of the Ras-Raf-MEK and PI3K/Akt pathways. (A) Jurkat (Mock) and Jurkat ( $\Delta$ BRAP2) cells were lysed, and BRAP2 and $\beta$-actin were detected via western blotting. (B) Jurkat (Mock) and Jurkat ( $\triangle$ BRAP2) cells were counted at the indicated times. Each bar denotes the standard deviation $(\mathrm{n}=3)$. ${ }^{* *} \mathrm{P}<0.01,{ }^{* * *} \mathrm{P}<0.001$ vs. 0 h samples. (C) Jurkat (Mock) and Jurkat ( $\triangle$ BRAP2) cells were lysed and $\mathrm{p}-\mathrm{Raf}, \mathrm{Raf}, \mathrm{p}-\mathrm{MEK}$, MEK, p-ERK, ERK, p-Akt, Akt, KSR and $\beta$-actin were detected via western blotting. (D) Jurkat (Mock) and Jurkat ( $\Delta$ BRAP2) cells were incubated with $5 \mu \mathrm{M}$ Ras inhibitor tipifarnib or $10 \mu \mathrm{M}$ pan-Raf inhibitor LY3009120 for the indicated times. The cells were lysed and p-Raf, Raf, p-MEK, MEK, p-ERK, ERK, and $\beta$-actin were detected via western blotting. The relative densities of the $\mathrm{p}$-Raf, $\mathrm{p}$-MEK, $\mathrm{p}$-ERK bands were estimated and normalized to the $\beta$-actin band. (E) Jurkat (Mock) and Jurkat ( $\Delta$ BRAP2) cells were incubated with $30 \mu \mathrm{M}$ PI3K inhibitor LY294002 for the indicated times. The cells were lysed and p-Akt, Akt and $\beta$-actin were detected via western blotting. The relative density of the p-Akt band was estimated and normalized to the $\beta$-actin band. BRAP2, breast cancer susceptibility gene 1-associated protein 2; p-phosphorylated; KSR, kinase suppressor of Ras. 

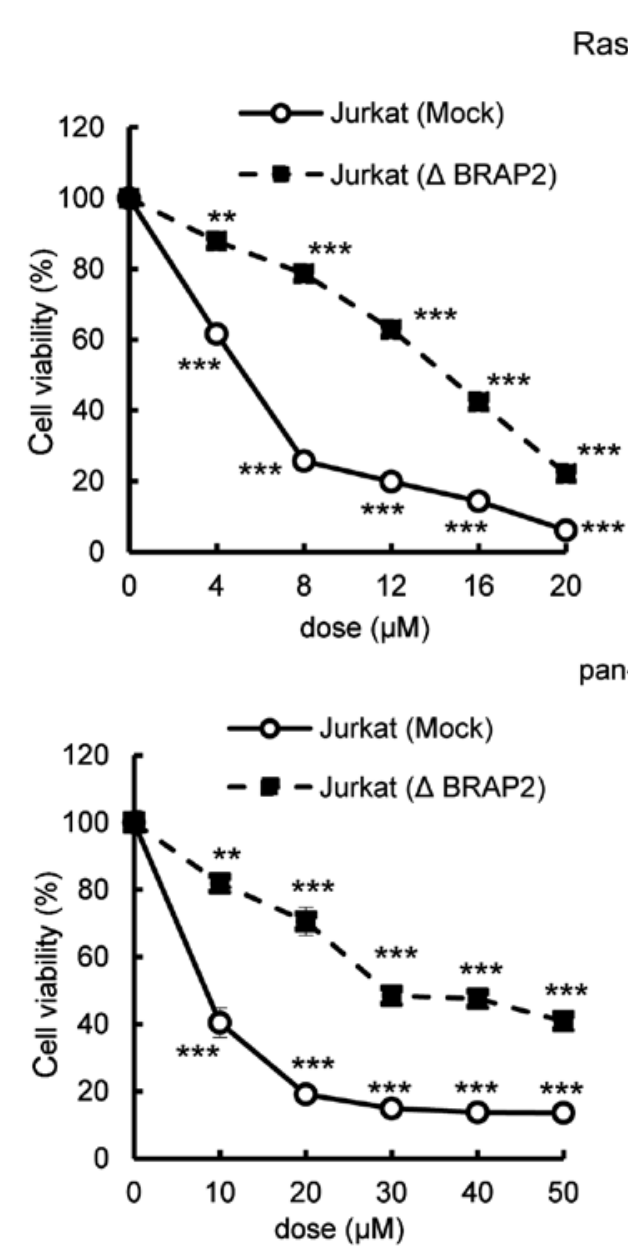

pan-Raf inhibitor

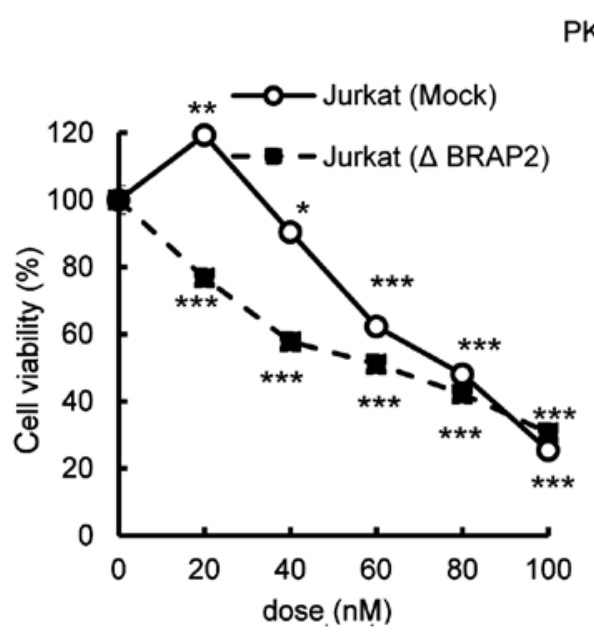

Figure 2. Loss of BRAP2 suppresses the cytotoxicity of Ras and pan-Raf inhibitors. Jurkat (Mock) and Jurkat ( $\triangle$ BRAP2) cells were incubated with the indicated doses of Ras inhibitor, pan-Raf inhibitor or PKC inhibitor staurosporine for $24 \mathrm{~h}$. Cell proliferation was estimated using the MTT assay. The data are presented in comparison to non-additive control cells. Each bar denotes the standard deviation $(\mathrm{n}=3) .{ }^{*} \mathrm{P}<0.05,{ }^{* * *} \mathrm{P}<0.01,{ }^{* * *} \mathrm{P}<0.001$ vs. untreated cells. BRAP2, breast cancer susceptibility gene 1-associated protein 2; PKC, protein kinase $\mathrm{C}$.

an MTT assay of the inhibitor cytotoxicity. We found that the loss of BRAP2 suppressed the cytotoxicities of the Ras and pan-Raf inhibitors, but not the cytotoxicity of staurosporine, a PKC inhibitor, not targeting the Ras-Raf-MEK pathway or the PI3K/Akt pathway (Fig. 2) (32,33).
Since the MTT assay measures mitochondrial enzyme activity, it cannot determine whether each inhibitor induces cell death. However, cell death is usually accompanied by DNA fragmentation. Therefore, we investigated whether each inhibitor induces cell death by staining DNA with PI and detecting the subG1 phase using flow cytometry $(34,35)$. We found that the loss of BRAP2 suppressed DNA fragmentation by the Ras and pan-Raf inhibitors but not by the PKC inhibitor (Fig. 3). We next examined the influence of BRAP2 loss on apoptosis. During apoptosis, cleaved caspase-3 appears, so cleaved caspase-3 is often used as a marker of apoptosis (36). Therefore, to determine whether each inhibitor induce apoptosis in our present experiments, we evaluated cleaved caspase- 3 by western blotting. The Ras and Pan-Raf inhibitors increased the level of cleaved caspase-3 in Jurkat (Mock) cells, but cleaved caspase-3 was not observed in the Jurkat ( $\triangle$ BRAP2) cells following treatment with these inhibitors. BRAP2. On the other hand, cleaved caspase-3 was present in both Jurkat (Mock) and Jurkat ( $\triangle$ BRAP2) cells treated with the PKC inhibitor (Fig. 4).

Inhibition of the Ras-Raf-MEK pathway by a Ras inhibitor was involved in apoptosis. In order to investigate whether the Ras inhibitor used in this study induces apoptosis through the Ras-Raf-MEK pathway, the cells were subjected to co-treatment with the Ras inhibitor and the activator PMA. As shown in Fig. 5A, the phosphorylations of Raf, MEK, and ERK were inhibited by Ras inhibitor treatment alone but were restored by co-treatment with PMA and the Ras inhibitor (Fig. 5A). In addition, we examined whether PMA would suppress the Ras inhibitor-induced apoptosis. We found that Ras inhibitor treatment increased the levels of subG1-phase cells and cleaved caspase-3, while co-treatment with the Ras inhibitor and PMA suppressed the increase in subG1 and cleaved caspase-3 (Fig. 5B and C). This demonstrated that the Ras inhibitor induced apoptosis through the Ras-Raf-MEK pathway.

Loss of BRAP2 suppressed cell cycle arrest by a PI3K inhibitor. Next, we evaluated the involvement of BRAP2 in the PI3K/Akt pathway. An MTT assay was used to examine whether the loss of BRAP2 changed the susceptibility of cells to PI3K inhibitor-induced cell death. However, the results showed that the presence or absence of BRAP2 expression had no effect on the susceptibility of cells to PI3K inhibitor-induced cell death (Fig. 6A). Therefore, we further investigated the effect of the PI3K inhibitor on the cell cycle. Unlike the Ras and Pan-Raf inhibitors, the PI3K inhibitor increased the G1 phase cells in Jurkat (Mock) cells, and the loss of BRAP2 suppressed the increase in the G1 phase (Fig. 6B). Finally, we assessed the potential inhibition of cell proliferation by the PI3K inhibitor and found that the PI3K inhibitor did indeed inhibit the proliferation of Jurkat (Mock) cells, and the BRAP2 deletion suppressed this inhibition (Fig. 6C).

BRAP2 expression was similar among various leukemia cells. Finally, we investigated the BRAP2 expression levels in other leukemia cell lines by western blotting. The levels of BRAP2 protein expression in human monocytic THP-1 cells, human B-cell lymphoma BALL-1 cells, human acute promyelocytic HL-60 cells, and human acute T-lymphoblastic MOLT-4F 

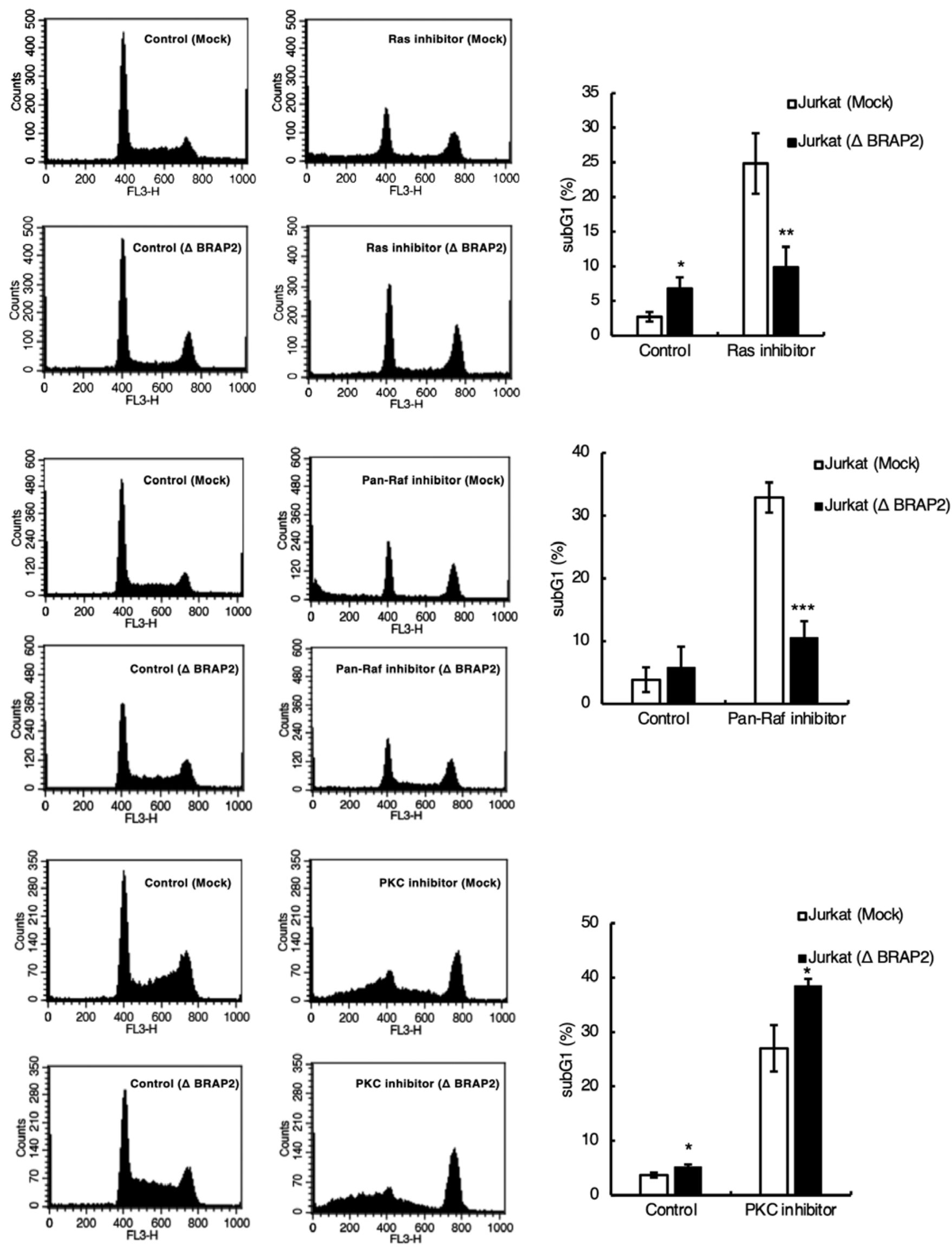

Figure 3. Loss of BRAP2 suppresses DNA fragmentation by the Ras and pan-Raf inhibitors. Jurkat (Mock) and Jurkat ( $\Delta$ BRAP2) cells were incubated with $5 \mu \mathrm{M}$ Ras inhibitor, $10 \mu \mathrm{M}$ pan-Raf inhibitor or $60 \mathrm{nM}$ PKC inhibitor for $24 \mathrm{~h}$. SubG1 phase was detected by flow cytometric analysis with PI staining, as described in the Materials and methods section. Representative histograms of one of three independent measurements are shown. The bar graph shows the percentages of cells in the sub-G1 phase. Each bar denotes the standard deviation $(\mathrm{n}=3)$. ${ }^{*} \mathrm{P}<0.05,{ }^{* * *} \mathrm{P}<0.01,{ }^{* * *} \mathrm{P}<0.001$ vs. mock cells. BRAP2, breast cancer susceptibility gene 1-associated protein 2; PKC, protein kinase C. 


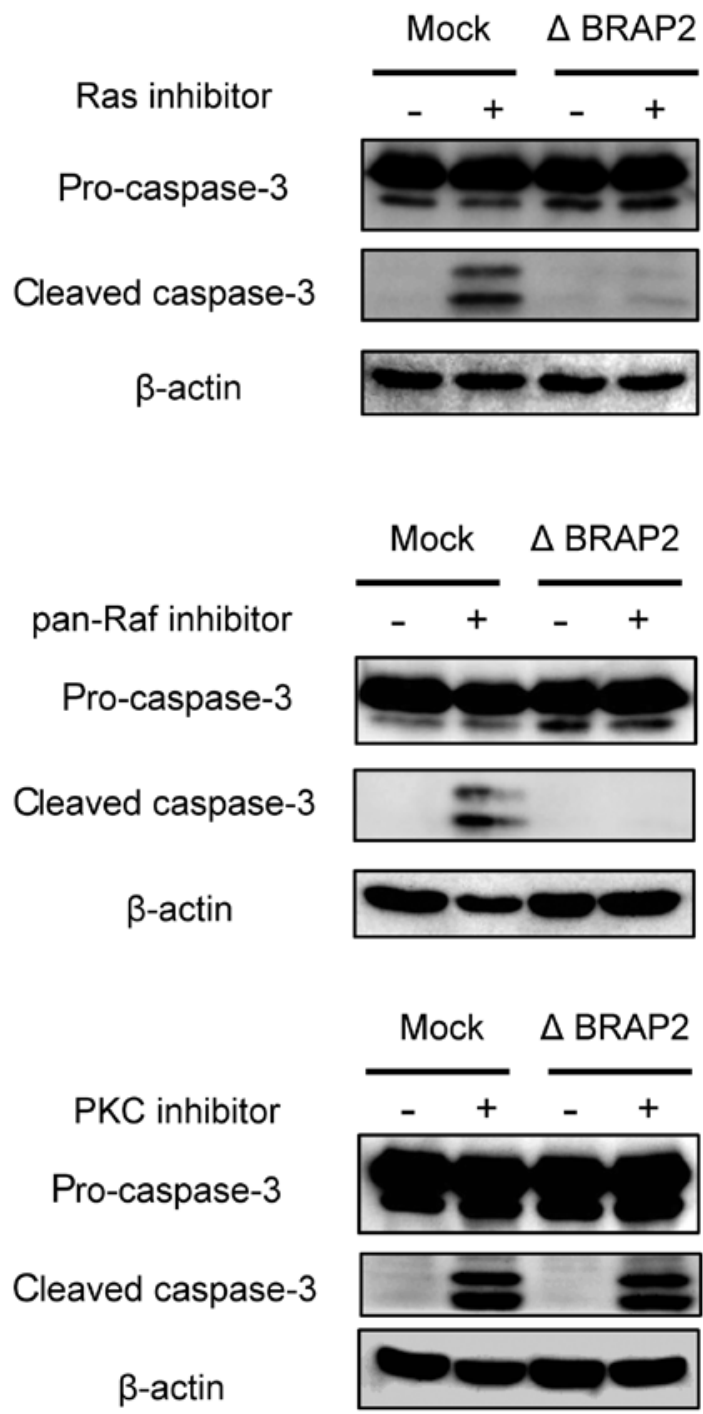

Figure 4. Loss of BRAP2 suppresses caspase- 3 cleavage by the Ras and pan-Raf inhibitors. Jurkat (Mock) and Jurkat ( $\triangle$ BRAP2) cells were incubated with $5 \mu \mathrm{M}$ Ras inhibitor, $10 \mu \mathrm{M}$ pan-Raf inhibitor, or $60 \mathrm{nM} \mathrm{PKC}$ inhibitor for $24 \mathrm{~h}$. The cells were lysed, and caspase- 3 and $\beta$-actin expression levels were detected via western blotting. BRAP2, breast cancer susceptibility gene 1-associated protein 2 .

cells were almost the same as those in human lymphoid helper T-cell line Jurkat cells (Fig. 7).

\section{Discussion}

Because BRAP2 is required for homeostasis, the mutation or deletion of BRAP2 may cause carcinogenesis (37). In addition, BRAP2 has been shown to interact with regulators of the Ras-Raf-MEK and PI3K/Akt pathways, thereby contributing to carcinogenesis $(26,27)$. These reports suggest that BRAP2 plays a role in carcinogenesis by regulating the Ras-Raf-MEK and PI3K/Akt pathways; however, the relationships between BRAP2 and these pathways in cancer are still unclear. Therefore, in this study, to clarify the role of BRAP2 on the Ras-Raf-MEK and PI3K/Akt pathways, we deleted BRAP2. We found that the deletion suppressed apoptosis by a Ras inhibitor and a pan-Raf inhibitor and inhibited the cell cycle arrest by a PI3K inhibitor.
After generating BRAP2-deficient cells, we conducted a western blot analysis to evaluate changes in the Ras-Raf-MEK and the PI3K/Akt pathways in the normal state, since BRAP2 has been reported to be involved in both pathways $(26,27)$. Interestingly, BRAP2 deletion increased the phosphorylation levels of Raf and MEK (Fig. 1C). KSR is a scaffold protein and enhances the Ras-Raf-MEK pathway (8-10). Because BRAP2 inhibits KSR and thereby impedes the signal transduction from Raf to MEK (26), we considered that BRAP2 deletion may increase the phosphorylation levels of Raf and MEK. In our present experiments, it was also of interest that BRAP2 deletion caused an increase in the phosphorylation level of Akt (Fig. 1C). A recent report showed that BRAP2 knockdown led to an increase in the phosphorylation levels of Akt and mTOR in glioma cells (38), and our result was consistent with that report. BRAP2 binds PHLPP1 $(27,39)$ which is involved in Akt activation $(28,29)$, and it has been suggested that BRAP2 suppresses Akt through PHLPP1 to promote apoptosis induction (39). Therefore, we thought that the phosphorylation level of Akt may have been increased because BRAP2 deletion could not suppress Akt through PHLPP1. Taken together, these results suggest that BRAP2 negatively regulates the Ras-Raf-MEK and PI3K/Akt pathways through binding partners in a normal state. BRAP2 deletion also attenuated the Ras inhibitor-mediated and pan-Raf inhibitor-mediated apoptosis (Figs. 3 and 4). KSR acts as a scaffold to bind Raf, MEK, and ERK, but KSR also possesses an intrinsic Raf activating mechanism independent of Ras (40). That is, KSR and Raf heterodimerization directly activate the Raf-MEK-ERK pathway. The Ras inhibitor used in this study, inhibits farnesyl transferase and target the region upstream of KSR, while the pan-Raf inhibitor LY3009120 inhibits Raf dimerization by the Ras signal, which is also upstream of KSR (41). Therefore, we considered that BRAP2 deletion increases the scaffolding and Raf-activating ability of KSR and further activates MEK and ERK independently of Ras $(7,26,40)$, which is in agreement with our present findings.

If the inhibitors used in this study targeted further downstream than KSR, the influence of BRAP2 deletion might be unaffected. For instance, the MEK inhibitor U-0126 is an inhibitor further downstream than KSR. However, U-0126 nonspecifically inhibited Akt as well as ERK phosphorylation at concentrations that induced cell death (data not shown), and thus U-0126 treatment could not be used to confirm our hypothesis. In light of all the above, we conclude that BRAP2 deletion attenuated Ras and pan-Raf inhibitor activity in this study.

In the PI3K/Akt pathway, the PI3K inhibitor blocked the level of p-Akt within $24 \mathrm{~h}$; however, it was found to restore p-Akt within $48 \mathrm{~h}$ in the presence of BRAP2. Deletion of BRAP2 suppressed the inhibition of Akt phosphorylation by the PI3K inhibitor for $48 \mathrm{~h}$ (Fig. 1E). BRAP2 was found to bind to not only KSR, but other proteins as well. One of them is PHLPP, which controls Akt phosphorylation $(28,29)$. Also, the PI3K/Akt pathway is frequently overactive in T-ALL (42). Thus, we predicted that BRAP2 can regulate the level of p-Akt within $24 \mathrm{~h}$, but, due to the abnormal activation of the PI3K/Akt pathway, the decreased levels of p-Akt were restored within $48 \mathrm{~h}$. Similarly, it is considered that the PI3K inhibitor induced cell growth inhibition rather than apoptosis, unlike the Ras-Raf-MEK pathway 


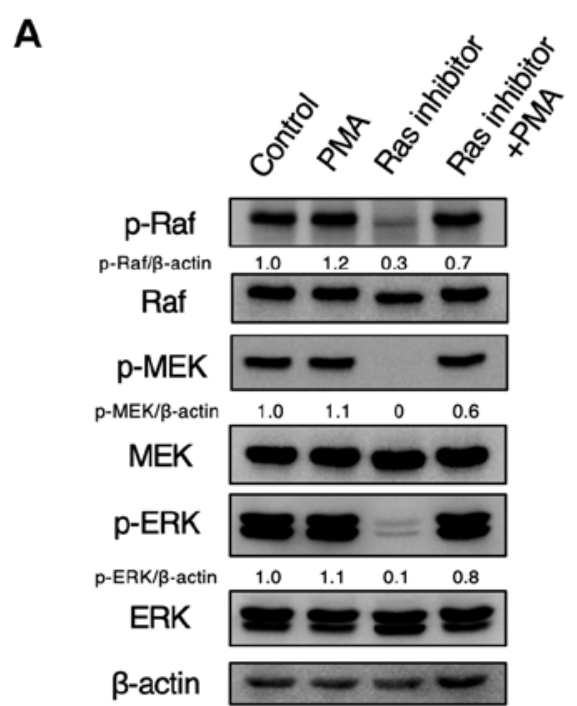

B
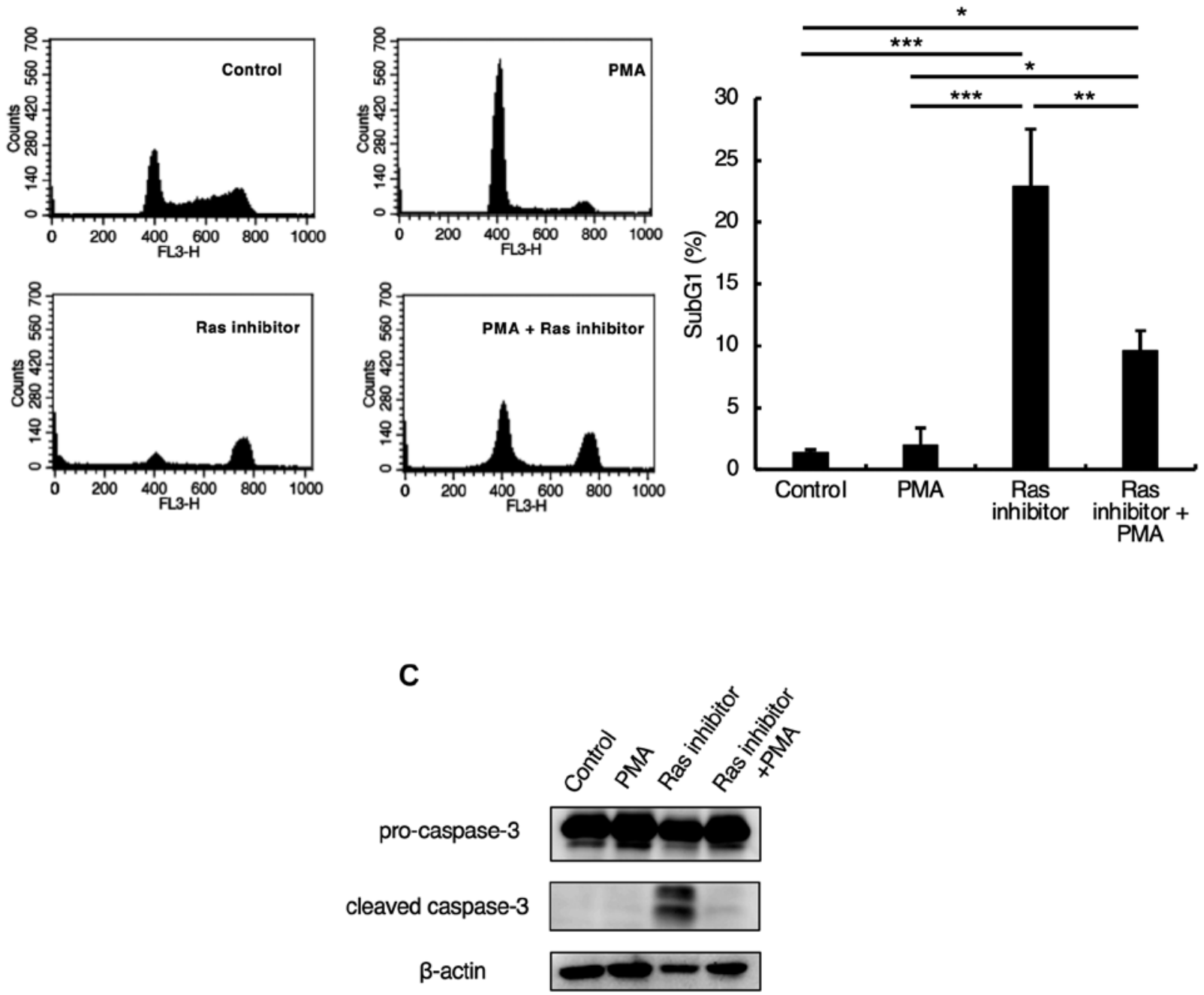

Figure 5. Inhibition of the Ras-Raf-MEK pathway by a Ras inhibitor influences apoptosis. (A) Jurkat cells were preincubated with $100 \mathrm{nM}$ PMA for $1 \mathrm{~h}$ and incubated with $5 \mu \mathrm{M}$ Ras inhibitor for $24 \mathrm{~h}$. Cells were lysed and p-Raf, Raf, p-MEK, MEK, p-ERK, ERK, and $\beta$-actin were detected by western blotting. The relative density of the p-Raf, p-MEK, p-ERK bands were estimated and normalized by $\beta$-actin band. (B) Jurkat cells were preincubated with $100 \mathrm{nM}$ PMA for $1 \mathrm{~h}$ and incubated with $5 \mu \mathrm{M}$ Ras inhibitor for $24 \mathrm{~h}$. SubG1 phase was detected by flow cytometric analysis with PI staining, as described in the Materials and methods section. Representative histograms of one of three independent measurements are shown. The bar graph shows the percentages of cells in the sub-G1 phase. Each bar denotes the standard deviation $(\mathrm{n}=3)$. ${ }^{*} \mathrm{P}<0.05,{ }^{* *} \mathrm{P}<0.01,{ }^{* * *} \mathrm{P}<0.001$. (C) Jurkat cells were preincubated with $100 \mathrm{nM}$ PMA for $1 \mathrm{~h}$ and incubated with $5 \mu \mathrm{M}$ Ras inhibitor for $24 \mathrm{~h}$. The cells were lysed, and caspase-3 and $\beta$-actin expression levels were detected via western blotting. BRAP2, breast cancer susceptibility gene 1-associated protein 2; p-, phosphorylated-; PMA, phorbol 12-myristate 13-acetate. 

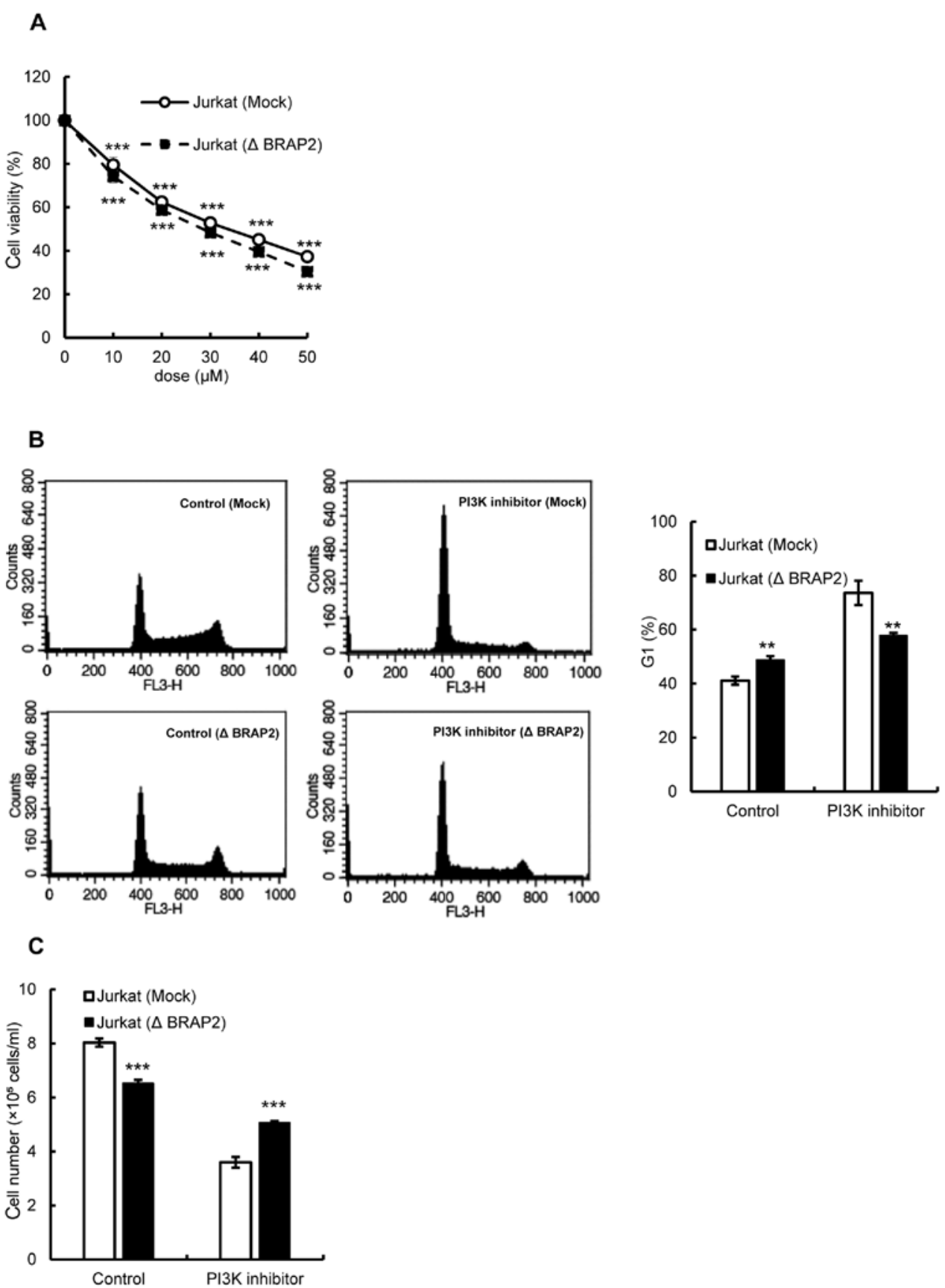

Figure 6. Loss of BRAP2 suppresses cell cycle arrest caused by PI3K inhibitor. (A) Jurkat (Mock) and Jurkat ( $\Delta$ BRAP2) cells were incubated with the indicated doses of PI3K inhibitor for $24 \mathrm{~h}$. Cell proliferation was estimated using the MTT assay. The data are presented in comparison to nonadditive control cells. Each bar denotes the standard deviation $(\mathrm{n}=3) .{ }^{* * *} \mathrm{P}<0.001$ vs. untreated cells. (B) Jurkat (Mock) and Jurkat $(\Delta \mathrm{BRAP})$ cells were incubated with $30 \mu \mathrm{M}$ PI3K inhibitor for $24 \mathrm{~h}$. G1 phase was detected via flow cytometric analysis with PI staining, as described in the Materials and methods section. Representative histograms of one of three independent measurements are shown. The bar graph shows the percentages of cells in the G1 phase. Each bar denotes the standard deviation ( $\mathrm{n}=3) .{ }^{* *} \mathrm{P}<0.01$ vs. mock cells. (C) Jurkat (Mock) and Jurkat $(\Delta \mathrm{BRAP} 2)$ cells were incubated with $30 \mu \mathrm{M}$ PI $3 \mathrm{~K}$ inhibitor for $48 \mathrm{~h}$. The cells were counted at 48 h. Each bar denotes the standard deviation $(n=3) .{ }^{* * * *} \mathrm{P}<0.001$ vs. mock cells. BRAP2, breast cancer susceptibility gene 1-associated protein 2 .

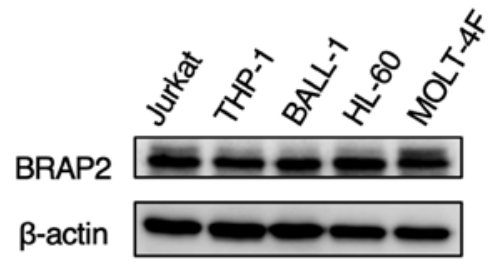

Figure 7. BRAP2 expression is similar among various leukemia cells. Jurkat, THP-1, BALL-1, HL-60 and MOLT-4F cells were lysed, and BRAP2 and $\beta$-actin were detected via western blotting. BRAP2, breast cancer susceptibility gene 1-associated protein 2 . inhibitors, because of the abnormal activation of the PI3K/Akt pathway in Jurkat cells (Fig. 6C). It was reported that the Jurkat cell line used in this study is deficient in phosphatase and tensin homolog deleted on chromosome 10 (PTEN) (43). PTEN is a tumor suppressor and regulates the survival of T-cells through the PI3K/Akt pathway. Because Jurkat cells are deficient in PTEN, apoptosis is suppressed $(43,44)$. Therefore, we predicted that the PI3K inhibitor inhibited cell proliferation, not apoptosis. However, the interaction between BRAP2 and the mutated protein or pathway is still unclear and needs further study. 
In summary, the present study focused on signal transduction, apoptosis, and cell proliferation to clarify the role of BRAP2 on the Ras-Raf-MEK and PI3K/Akt pathways. The results showed that BRAP2 induces apoptosis and cell growth inhibition against Jurkat cells by negatively regulating the Ras-Raf-MEK and PI3K/Akt pathways. These pathways are important targets in cancer treatment, and BRAP2 negatively regulates them. Moreover, we found that BRAP2 expression was mostly similar among various leukemia cells, suggesting that BRAP2 regulates the Ras-Raf-MEK and PI3K/Akt pathways in different leukemia cell lines (Fig. 7). Moreover, in a recent report, a reduction in BRAP2 expression was shown to promote cancer cell proliferation both in vitro and in vivo (38). Further studies are warranted to investigate the BRAP2 expression and cytotoxicities compared with normal lymphocytes for cancer cell progression, and we will examine in the future study. Therefore, the development of a drug that enhances the function of BRAP2 would be a new approach in cancer treatment.

\section{Acknowledgements}

Not applicable.

\section{Funding}

No funding was received.

\section{Availability of data and materials}

The datasets used and/or analyzed during the current study are available from the corresponding author on reasonable request.

\section{Authors' contributions}

YN and TS designed the research. HS performed the experiments. YN and HS drafted the manuscript and analyzed data. IS, TS and HS interpreted data and revised the manuscript. YN and HS confirm the authenticity of all the raw data. All authors read and approved the final manuscript.

\section{Ethics approval and consent to participate}

Not applicable.

\section{Patient consent for publication}

Not applicable.

\section{Competing interests}

The authors declare that they have no competing interests.

\section{References}

1. Raman M, Chen W and Cobb MH: Differential regulation and properties of MAPKs. Oncogene 26: 3100-3112, 2007.

2. Brunet A, Roux D, Lenormand P, Dowd S, Keyse S and Pouysségur J: Nuclear translocation of p42/p44 mitogenactivated protein kinase is required for growth factor-induced gene expression and cell cycle entry. EMBO J 18: 664-674, 1999.
3. Roberts PJ and Der CJ: Targeting the Raf-MEK-ERK mitogen-activated protein kinase cascade for the treatment of cancer. Oncogene 26: 3291-3310, 2007.

4. Downward J: Targeting RAS signalling pathways in cancer therapy. Nat Rev Cancer 3: 11-22, 2003.

5. Corcoran RB, Ebi H, Turke AB, Coffee EM, Nishino M, Cogdill AP, Brown RD, Della Pelle P, Dias-Santagata D, Hung KE, et al: EGFR-mediated re-activation of MAPK signaling contributes to insensitivity of BRAF mutant colorectal cancers to RAF inhibition with vemurafenib. Cancer Discov 2: 227-235, 2012.

6. Misale S, Yaeger R, Hobor S, Scala E, Janakiraman M, Liska D, Valtorta E, Schiavo R, Buscarino M, Siravegna G, et al: Emergence of KRAS mutations and acquired resistance to anti-EGFR therapy in colorectal cancer. Nature 28: 532-536, 2012.

7. Vakana E, Pratt S, Blosser W, Dowless M, Simpson N, Yuan XJ, Jaken S, Manro J, Stephens J, Zhang Y, et al: LY3009120, a pan RAF inhibitor, has significant anti-tumor activity in BRAF and KRAS mutant preclinical models of colorectal cancer. Oncotarget 8: 9251-9266, 2017.

8. Therrien M, Michaud NR, Rubin GM and Morrison DK: KSR modulates signal propagation within the MAPK cascade. Gene Dev 10: 2684-2695, 1996.

9. Kortum RL and Lewis RE: The molecular scaffold KSR1 regulates the proliferative and oncogenic potential of cells. Mol Cell Biol 24: 4407-4416, 2004.

10. Razidlo GL, Kortum RL, Haferbier JL and Lewis RE: Phosphorylation regulates KSR1 stability, ERK activation, and cell proliferation. J Biol Chem 279: 47808-47814, 2004.

11. Wang L, Jiang CF, Li DM, Ge X, Shi ZM, Li CY, Liu X, Yin Y, Zhen L, Liu LZ and Jiang BH: MicroRNA-497 inhibits tumor growth and increases chemosensitivity to 5-fluorouracil treatment by targeting KSR1. Oncotarget 7: 2660-2671, 2016.

12. Zhou L, Lyons-Rimmer J, Ammoun S, Muller J, Lasonder E, Sharma V, Ercolano E, Hilton D, Taiwo I, Barczyk M and Hanemann CO: The scaffold protein KSR1, a novel therapeutic target for the treatment of merlin-deficient tumors. Oncogene 35: 3443-3453, 2016.

13. Posch C, Moslehi H, Feeney L, Green GA, Ebaee A, Feichtenschlager V, Chong K, Peng L, Dimon MT, Phillips T, et al: Combined targeting of MEK and PI3K/mTOR effector pathways is necessary to effectively inhibit NRAS mutant melanoma in vitro and in vivo. Proc Natl Acad Sci USA 110: 4015-4020, 2013.

14. Mendoza MC, Er EE and Blenis J: The Ras-ERK and PI3K-mTOR pathways: Cross-talk and compensation. Trends Biochem Sci 36: 320-328, 2011.

15. Schult C, Dahlhaus M, Ruck S, Sawitzky M, Amoroso F, Lange S, Etro D, Glass A, Fuellen G, Boldt S, et al: The multikinase inhibitor Sorafenib displays significant antiproliferative effects and induces apoptosis via caspase 3,7 and PARP in B- and T-lymphoblastic cells. BMC Cancer 10: 560, 2010.

16. Coloff JL, Mason EF, Altman BJ, Gerriets VA, Liu T, Nichols AN, Zhao Y, Wofford JA, Jacobs SR, Ilkayeva O, et al: Akt requires glucose metabolism to suppress puma expression and prevent apoptosis of leukemic T cells. J Biol Chem 286: 5921-5933, 2011.

17. Huang Y, Wu S, Zhang Y, Wang L and Guo Y: Antitumor effect of triptolide in T-cell lymphoblastic lymphoma by inhibiting cell viability, invasion, and epithelial-mesenchymal transition via regulating the $\mathrm{PI} 3 \mathrm{~K} / \mathrm{AKT} / \mathrm{mTOR}$ pathway. Onco Targets Ther 11: 769-779, 2018.

18. Kiessling MK, Curioni-Fontecedro A, Samaras P, Atrott K, Cosin-Roger J, Lang S, Scharl M and Rogler G: Mutant HRAS as novel target for MEK and mTOR inhibitors. Oncotarget 6: 42183-42196, 2015.

19. Rodriguez-Viciana P, Warne PH, Dhand R, Vanhaesebroeck B, Gout I, Fry MJ, Waterfield MD and Downward J: Phosphatidylinositol-3-OH kinase as a direct target of Ras. Nature 370: 527-532, 1994.

20. Diaz-Flores E and Shannon K: Targeting oncogenic ras. Gene Dev 21: 1989-1992, 2007.

21. Li S, Ku CY, Farmer AA, Cong YS, Chen CF and Lee WH: Identification of a novel cytoplasmic protein that specifically binds to nuclear localization signal motifs. J Biol Chem 273: 6183-6189, 1998

22. Asada M, Ohmi K, Delia D, Enosawa S, Suzuki S, You A, Suzuki H and Mizutani S: Brap2 functions as a cytoplasmic retention protein for $\mathrm{p} 21$ during monocyte differentiation. Mol Cell Biol 24: 8236-8243, 2004. 
23. Takashima O, Tsuruta F, Kigoshi Y, Nakamura S, Kim J, Katoh MC, Fukuda T, Irie K and Chiba T: Brap2 regulates temporal control of NF- $\kappa \mathrm{B}$ localization mediated by inflammatory response. PLoS One 8: e58911, 2013.

24. Ozaki K, Sato H, Inoue K, Tsunoda T, Sakata Y, Mizuno H, Lin TH, Miyamoto Y, Aoki A, Onouchi Y, et al: SNPs in BRAP associated with risk of myocardial infarction in Asian populations. Nat Genet 41: 329-333, 2009.

25. Liao YC, Wang YS, Guo YC, Ozaki K, Tanaka T, Lin HF, Chang MH, Chen KC, Yu ML, Sheu SH and Juo SH: BRAP activates inflammatory cascades and increases the risk for carotid atherosclerosis. Mol Med 17: 1065-1074, 2011.

26. Matheny SA, Chen C, Kortum RL, Razidlo GL, Lewis RE and White MA: Ras regulates assembly of mitogenic signalling complexes through the effector protein IMP. Nature 427 : 256-260, 2004

27. Fatima S, Wagstaff KM, Loveland KL and Jans DA: Interactome of the negative regulator of nuclear import BRCA1-binding protein 2. Sci Rep 5: 9459, 2015.

28. Gao T, Furnari F and Newton AC: PHLPP: A phosphatase that directly dephosphorylates Akt, promotes apoptosis, and suppresses tumor growth. Mol Cell 18: 13-24, 2005.

29. Brognard J, Sierecki E, Gao T and Newton AC: PHLPP and a second isoform, PHLPP2, differentially attenuate the amplitude of Akt signaling by regulating distinct Akt isoforms. Mol Cell 25 917-931, 2007.

30. Ran FA, Hsu PD, Wright J, Agarwala V, Scott DA and Zhang F: Genome engineering using the CRISPR-Cas9 system. Nat Protoc 11: 2281-2308, 2013

31. Lanctot AA, Guo Y, Le Y, Edens BM, Nowakowski RS and Feng Y: Loss of brap results in premature G1/S phase transition and impeded neural progenitor differentiation. Cell Rep 20 1148-1160, 2017

32. Shi L, Weng XQ, Sheng Y,Wu J, Ding M and Cai X: Staurosporine enhances ATRA-induced granulocytic differentiation in human leukemia U937 cells via the MEK/ERK signaling pathway. Oncol Rep 36: 3072-3080, 2016.

33. Liu AH, Cao YN, Liu HT, Zhang WW, Liu Y, Shi TW, Jia GL and Wang XM: DIDS attenuates staurosporine-induced cardiomyocyte apoptosis by PI3K/Akt signaling pathway: Activation of eNOS/NO and inhibition of Bax translocation. Cell Physiol Biochem 22: 177-186, 2008.
34. Darzynkiewicz Z, Bruno S, Del Bino G, Gorczyca W, Hotz MA, Lassota $\mathrm{P}$ and Traganos F: Features of apoptotic cells measured by flow cytometry. Cytometry 13: 795-808, 1992.

35. Bedner E,Li X, Gorczyca W, Melamed MR and Darzynkiewicz Z Analysis of apoptosis by laser scanning cytometry. Cytometry 35 : 181-195, 1999.

36. Cohen GM: Caspase: The executioners of apoptosis. Biochem J 326: 1-16, 1997

37. Koon JC and Kubiseski TJ: Developmental arrest of caenorhabditis elegans BRAP-2 mutant oxidative stress is dependent on BRC-1. J Biol Chem 285: 13437-13443, 2010.

38. Wang B, Cao C, Liu X, He X, Zhuang H, Wang D and Chen B: BRCA1-associated protein inhibits glioma cell proliferation and migration and glioma stem cell self-renewal via the TGF- $\beta / \mathrm{PI} 3 \mathrm{~K} /$ AKT/mTOR signalling pathway. Cell Oncol (Dordr) 43: 223-235, 2020.

39. D'Amora DR, Hu Q, Pizzardi M and Kubiseski TJ: BRAP-2 promotes DNA damage induced germline apoptosis in $\mathrm{C}$. elegans through the regulation of SKN-1 and AKT-1. Cell Death Differ 25: 1276-1288, 2018.

40. Rajakulendran T, Sahmi M, Lefraancois M, Sicheri F and Therrien M: A dimerization-dependent mechanism drives RAF catalytic activation. Nature 461: 542-545, 2009.

41. Peng SB, Henry JR, Kaufman MD, Lu WP, Smith BD, Vogeti S, Rutkoski TJ, Wise S, Chun L, Zhang Y, et al: Inhibition of RAF isoforms and active dimers by LY3009120 leads to anti-tumor activities in RAS or BRAF mutant cancers. Cancer Cell 28: 384-398, 2015

42. Zhao WL: Targeted therapy in T-cell malignancies: Dysregulation of the cellular signaling pathways. Leukemia 24 $13-21,2010$

43. Xu Z, Stokoe D, Kane LP and Weiss A: The inducible expression of the tumor suppressor gene PTEN promotes apoptosis and decreases cell size by inhibiting the PI3K/Akt pathway in Jurkat T cells. Cell Growth Differ 13: 285-296, 2002.

44. Wang Z, Gjörloff-Wingren A, Saxena M, Pathan N, Reed JC and Mustelin T: The tumor suppressor PTEN regulates T cell survival and antigen receptor signaling by acting as a phosphatidylinositol 3-phosphatase. J Immunol 164: 1934-1939, 2000. 